\title{
Psicooncología
}

ISSN: 1696-7240

\section{La relevancia de la flexibilidad psicológica en el contexto del cáncer: una revisión de la literatura}

\author{
Aldo Aguirre-Camacho ${ }^{1 *}$; Bernardo Moreno-Jiménez ${ }^{2}$
}

Recibido: 20 de marzo de 2017 / Aceptado: 14 de abril de 2017

Resumen. La flexibilidad psicológica ha sido relacionada con diversos indicadores positivos en individuos con problemas de salud mental y enfermedades crónicas, y ha sido identificada como un importante mecanismo de cambio psicoterapéutico. Sin embargo, la atención prestada a la flexibilidad psicológica en la psicooncología ha sido limitada, a pesar de su potencial relación con el distrés relacionado con el cáncer. El objetivo de la presente revisión descriptiva fue reunir los estudios que han examinado la asociación entre la flexibilidad psicológica y distintos indicadores de bienestar en pacientes con cáncer, o evaluado el impacto de intervenciones destinadas a incrementar el nivel de flexibilidad psicológica en pacientes con cáncer. A pesar del bajo número de estudios, los resultados demuestran de forma consistente que la flexibilidad psicológica está asociada a altos niveles de bienestar en pacientes con cáncer, incluso en presencia de deterioro físico. Para finalizar, se presentan algunas implicaciones para la práctica clínica en psico-oncología.

Palabras clave: Flexibilidad psicológica; evitación experiencial; Terapia de Aceptación y Compromiso; ACT; cáncer.

\section{[en] The relevance of psychological flexibility in the context of cancer: a review of the literature}

\begin{abstract}
Psychological flexibility has been related to a wide number of positive outcomes among individuals with mental health problems and chronic illnesses, and it has been identified as an important mechanism of change in psychotherapy. However, the attention given to psychological flexibility within psycho-oncology has been quite limited, even though it may offer new alternatives in the treatment of cancer-related distress. The objective of the present descriptive review was to bring together the studies that have examined the association between psychological flexibility and outcomes among cancer patients, or have carried out interventions aiming to increase the level of psychological flexibility among cancer patients. Even though the research base in this area is still small, the findings consistently show that higher levels of psychological flexibility are associated with increased levels of wellbeing among cancer patients, even in the presence of health deterioration. Implications for clinical practice in psycho-oncology are discussed.
\end{abstract}

Keywords: Psychological flexibility; experiential avoidance; Aceptance and Commitment Therapy; ACT; cancer.

1 Universidad Autónoma de Madrid.

aldo.aguirre.c@gmail.com

2 Universidad Autónoma de Madrid.

bernardo.moreno@uam.es

* Dirección de correspondencia: Aldo Aguirre-Camacho. Universidad Autónoma de Madrid, Ciudad Universitaria de Cantoblanco, Facultad de Psicología, Calle Ivan Pavlov 6, 28049 Madrid, España. 
Sumario. 1. Planteamiento del problema 2. Desarrollo del tema 2.1. El cáncer en los medios de comunicación 2.2. Cáncer, fusión cognitiva, y evitación experiencial 2.3. Estudios sobre flexibilidad psicológica y cáncer 3. Discusión e implicaciones para la práctica clínica 4. Referencias bibliográficas.

Cómo citar: Aguirre-Camacho A, Moreno-Jiménez B. La relevancia de la flexibilidad psicológica en el contexto del cáncer: una revisión de la literatura. Psicooncología 2017;14:11-22. DOI: 10.5209/ PSIC. 55808

\section{Planteamiento del problema}

El cáncer es una enfermedad con un alto potencial de causar un profundo impacto en la vida de quienes son diagnosticados. Tener cáncer va frecuentemente asociado a notables deterioros en el estado físico y funcional, ya sea por la enfermedad misma o por sus tratamientos. El impacto del cáncer sobre la salud puede además derivar en diversos factores de estrés, resultantes por ejemplo de las interrupciones en las actividades sociales familiares y ocupacionales, de posibles problemas económicos, y del sufrimiento que también puede causar a seres queridos. Al mismo tiempo, las personas con cáncer deben lidiar con la incertidumbre relacionada con tener una enfermedad relativamente impredecible, así como con la posibilidad de sufrir una recurrencia o la muerte.

Un porcentaje considerable de las personas diagnosticadas con cáncer se ven afectadas por problemas psicológicos. Los resultados de un reciente metaanálisis ${ }^{(1)}$, basado estrictamente en estudios que hubieran utilizado entrevistas clínicas estructuradas, mostraron que la prevalencia combinada estimada de trastornos psiquiátricos en pacientes con cáncer alcanza el 32\% (IC 95\%: 27\% a $37 \%$ ). Además del impacto directo de la enfermedad, existe evidencia de que las reacciones emocionales y psicológicas al cáncer pudieran estar influenciadas por aspectos socioculturales ${ }^{(2)}$, como si el entendimiento de lo que implica tener cáncer hubiera pasado a formar parte de un subconsciente colectivo. Algunos estudios por ejemplo han mostrado que el cáncer es la enfermedad más temida, incluso más que enfermedades de peor pronóstico ${ }^{(3)}$. Se ha visto que las actitudes y los significados atribuidos al cáncer pueden tener un impacto notable sobre el bienestar emocional de las personas diagnosticadas ${ }^{(4)}$, y parece que dichas actitudes y significados comienzan a establecerse antes del diagnóstico ${ }^{(5)}$.

El objetivo del presente trabajo es examinar la relación entre la flexibilidad psicológica y el bienestar psicológico en pacientes con cáncer, mediante una revisión descriptiva de la literatura. La flexibilidad psicológica ha sido definida como la "capacidad de contactar plenamente con el momento presente y los pensamientos y sentimientos que contiene, sin necesidad de defensa, y dependiendo de la situación, cambiar o persistir en el comportamiento con el objetivo de alcanzar fines valiosos"(6). El término "flexibilidad psicológica" hace también referencia al modelo del mismo nombre, el cual constituye la base de la Terapia de Aceptación y Compromiso (ACT). En el comienzo, los términos aceptación y evitación experiencial eran los usados en lugar de flexibilidad e inflexibilidad psicológica, respectivamente, para hacer referencia de forma positiva y negativa al proceso general descrito por el modelo, conocido inicialmente como Modelo de Evitación Experiencial ${ }^{(6)}$. A pesar de que actualmente todos estos términos continúan siendo utilizados de forma indistinta, en el presente 
trabajo haremos uso de los términos más actualizados: flexibilidad e inflexibilidad psicológica. La descripción del Modelo de Flexibilidad Psicológica no forma parte de los objetivos de la presente revisión, pero puede encontrarse en otros trabajos ${ }^{(7)}$.

Durante los últimos años, el apoyo empírico al modelo de flexibilidad psicológica ha ido creciendo exponencialmente. Diversas investigaciones en las áreas de psicopatología y terapia de conducta han mostrado evidencia de que la inflexibilidad psicológica constituye una forma generalizada de vulnerabilidad psicológica ${ }^{(8)}$. Por ejemplo, se ha visto implicada en la etiología y mantenimiento de trastornos mentales, tales como el trastorno de ansiedad generalizada ${ }^{(9)}$, trastorno de estrés posttraumático ${ }^{(10)}, \mathrm{y}$ depresión ${ }^{(11)}$, y en problemas crónicos de salud, como por ejemplo en enfermedades cardiovasculares ${ }^{(12)}$ y esclerosis múltiple ${ }^{(13)}$. Sin embargo, la atención dedicada a la flexibilidad psicológica en el ámbito de la psico-oncología ha sido muy limitada, a pesar de que existen razones teóricas y estudios en áreas relacionadas que apuntan a que podría ser de gran relevancia para explicar diversos fenómenos en el contexto de un diagnóstico de cáncer ${ }^{(14)}$.

La presente revisión cuenta con varios apartados. Primeramente se realiza un breve recuento sobre la construcción social del cáncer en los medios de comunicación y su influencia en las creencias y significados atribuidos al cáncer. Se argumenta, desde un punto de vista teórico, que dichas creencias y significados podrían ser relevantes en el entendimiento de la relación entre inflexibilidad psicológica y el distrés relacionado con el cáncer. Seguidamente, tomando el modelo de flexibilidad psicológica como base, se describen una serie de procesos que podrían conducir hacia la inflexibilidad psicológica en el contexto del cáncer. Luego, se presentan los estudios identificados por la revisión, y para concluir se ofrecen algunas implicaciones para la práctica clínica en psico-oncología.

La búsqueda de los estudios consultados en el presente trabajo fue realizada a través de las plataformas PubMed, PsycINFO, Google Académico, y Psicodoc. Se realizó la búsqueda en español e inglés utilizando los términos "flexibilidad psicológica", "inflexibilidad psicológica", "evitación experiencial", y "cáncer" (en inglés: "psychological flexibility", "psychological inflexibility", "experiential avoidance" y "cancer"). Además, se revisaron las referencias de los artículos encontrados con el objetivo de identificar otros artículos que pudieran resultar relevantes. Se incluyeron estudios que han examinado el papel de la flexibilidad psicológica en relación a diferentes aspectos relacionados con el bienestar de pacientes con cáncer o que han evaluado el impacto de intervenciones destinadas a incrementar la flexibilidad psicológica en pacientes con cáncer.

\section{Desarrollo del tema}

\subsection{El cáncer en los medios de comunicación}

La atención brindada al cáncer en los medios de comunicación (e.g. revistas, programas de televisión, internet) comenzó a incrementarse notablemente a partir de las últimas décadas del pasado siglo ${ }^{(15,16)}$. Desde entonces, los medios de comunicación se han convertido en una importante fuente de información sobre el cáncer, especialmente tomando en cuenta que muchas personas dependen de los medios de comunicación para la obtención de información sobre la salud ${ }^{(17)}$. Se ha planteado que la amplia atención 
brindada al cáncer en los medios de comunicación ha contribuido a incrementar el nivel de conciencia sobre diversos aspectos relacionados con el cáncer ${ }^{(18)}$. En el caso específico del cáncer de mama, por ejemplo, se ha propuesto que la disminución de las tasas de mortalidad observadas en varias naciones industrializadas en las últimas dos décadas ha sido en gran medida el resultado de una mayor concienciación sobre la importancia de las prácticas de detección precoz ${ }^{(19)}$.

No obstante, algunos estudios han reportado que en no pocas ocasiones los medios de comunicación presentan información distorsionada y enfocada a las consecuencias negativas asociadas al cáncer. A modo general, por ejemplo, se ha visto que el cáncer es frecuentemente asociado con miedo y pérdida de control, representado como una enfermedad "casi inevitable" que "se desarrolla sigilosamente", y que "puede afectar a cualquiera en cualquier momento"(20). Hay quienes plantean que la gran atención brindada al cáncer en los medios de comunicación, aunque por lo general bien intencionada, ha traído consigo algunos efectos adversos ${ }^{(2)}$. Algunos estudios han sugerido que la atención focalizada en el cáncer podría alterar las percepciones de riesgo y desviar la atención de otras enfermedades más prevalentes y/o de peor pronóstico ${ }^{(16,21)}$. Por ejemplo, varios estudios han encontrado que el cáncer de mama es el cáncer más temido por las mujeres, a pesar de su relativamente buen pronóstico comparado con otros tipos de cáncer y otras enfermedades ${ }^{(2,22)}$. Además, la investigación en esta área ha mostrado que un gran número de mujeres sobreestiman el riesgo de ser diagnosticadas con cáncer de mama y el impacto del tratamiento, y mantienen creencias erróneas y fatalistas acerca de las posibles consecuencias de un diagnóstico ${ }^{(23)}$.

\subsection{Cáncer, fusión cognitiva, y evitación experiencial}

Recibir un diagnóstico de cáncer puede resultar una experiencia altamente angustiosa. Por una parte, el cáncer continúa siendo una enfermedad potencialmente letal, por otra, el diagnóstico puede también activar narrativas adquiridas tras los años de exposición a historias catastrofistas y sesgadas sobre el cáncer. En este sentido, la presencia de tales narrativas y la fusión cognitiva con las mismas podrían en conjunto derivar en inflexibilidad psicológica.

El término fusión cognitiva hace referencia a uno de los seis procesos que componen el modelo de flexibilidad psicológica ${ }^{(7)}$. De acuerdo con este modelo, la fusión cognitiva consiste en un proceso de dominio verbal a través del cual "los eventos verbales ejercen un fuerte control estimular sobre la conducta, con la

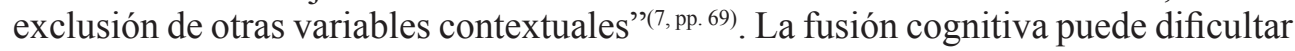
la distinción entre la experiencia directa y el contenido mental, y por lo tanto crear la impresión de que se responde a la experiencia directa cuando en realidad se responde al contenido mental. En el contexto de un diagnóstico de cáncer, por ejemplo, las reacciones emocionales (e.g. ansiedad elevada) podrían en gran medida resultar de la fusión cognitiva con narrativas catastrofistas (e.g. "el cáncer es lo peor"), y no solo de la experiencia directa con el cáncer. Además, la fusión cognitiva con determinadas reglas verbales (e.g. "lo malo debe evitarse" o "siempre hay que intentar sentirse bien") puede derivar en la evitación experiencial de pensamientos, sentimientos, o situaciones aversivas relacionadas con el cáncer. La evitación experiencial es otro de los seis procesos que componen el modelo de flexibilidad psicológica, y ha sido definida como "falta de voluntad de permanecer en contacto con eventos privados aversivos (e.g. emociones, pensamientos, sensaciones corporales), junto 
con esfuerzos deliberados para controlar o alterar la frecuencia, duración, o la forma de los eventos privados aversivos o de los acontecimientos o situaciones que los provocan"(7, pp. ${ }^{72}$. Sin embargo, se ha visto que el esfuerzo constante de evitar, controlar, o alterar los eventos privados parece adentrar a los individuos más profundamente en las experiencias que tratan de evitar, produciéndose aumentos a largo plazo en la gravedad y frecuencia de los eventos privados evitados, así como aumentos en los síntomas psicológicos ${ }^{(24)}$.

La evitación experiencial puede manifestarse en conductas topográficamente diferentes. En el contexto del cáncer, por ejemplo, las personas afectadas pueden optar por no mencionar la palabra cáncer, no mirarse las cicatrices producidas por las cirugías, obviar pensamientos/sentimientos relacionados con el cáncer, o no asistir a terapia de grupo. Sin embargo, conductas como estas pueden cumplir una función en común, en este caso la de regulación emocional mediante la supresión de eventos privados aversivos; es dicha función lo que define a la evitación experiencial.

\subsection{Estudios sobre flexibilidad psicológica y cáncer}

Las distintas investigaciones sobre flexibilidad psicológica que han sido realizadas en el contexto del cáncer pueden situarse dentro de dos grandes áreas. Por una parte, se encuentran los estudios que han evaluado la flexibilidad psicológica en el contexto de intervenciones terapéuticas, y por otra parte, se encuentran los estudios de tipo observacional que han examinado la relación entre la flexibilidad psicológica y diversos indicadores de bienestar de relevancia para las personas con cáncer.

La implicación de la flexibilidad psicológica en conductas relacionadas con el cáncer puede apreciarse incluso con anterioridad al diagnóstico. Existe evidencia de que la dificultad para entrar en contacto con eventos privados o situaciones aversivas relacionadas con el cáncer parece interferir con las conductas de prevención. Utilizando una muestra comunitaria de 87 mujeres sanas, Miller et al. ${ }^{(25)}$ mostraron que la flexibilidad psicológica moderó la relación entre el miedo al cáncer y la asistencia a exámenes de mamografía. Por una parte, se vio que las participantes con altos niveles de flexibilidad psicológica solían presentarse a sus exámenes de mamografías, debido a que no mostraban dificultad en hacer frente incluso a altos niveles de miedo o sentimientos aversivos relacionados con el cáncer. Por otra parte, las participantes con bajos niveles de flexibilidad psicológica y altos niveles de miedo también mostraron una alta tasa de asistencia a los exámenes de mamografía, debido a que el miedo las motivaba a reducir la incertidumbre sobre si tenían cáncer o no. Sin embargo, las participantes con bajos niveles de miedo y bajos niveles de flexibilidad psicológica mostraron una baja tasa de asistencia a los exámenes de mamografía, a pesar de las posibles consecuencias. Ello parece haber sido el resultado de la dificultad para entrar en contacto con eventos privados aversivos (e.g. miedo a recibir un resultado positivo) y las molestias físicas asociadas con la mamografía.

De forma congruente con estudios llevados a cabo en el contexto de otras enfermedades crónicas, algunos estudios realizados con personas con cáncer han mostrado que la flexibilidad psicológica está asociada a diversos indicadores de bienestar. Por ejemplo, en un estudio con 129 pacientes con cáncer, HulbertWilliams y Storey ${ }^{(26)}$ mostraron que la flexibilidad psicológica estuvo relacionada 
con niveles más altos de afecto positivo, calidad de vida, y beneficios asociados al cáncer, así como con menores niveles de afecto negativo, ansiedad, depresión, y estrés. Resultados similares fueron obtenidos por Montiel et al. ${ }^{(27)}$, quienes mostraron que la flexibilidad psicológica predijo menores niveles de ansiedad, depresión, y afecto negativo en una muestra de 64 mujeres con cáncer de mama al cabo de seis meses de seguimiento. Utilizando una muestra de 107 personas con diversos tipos de cáncer, Ciarrochi et al. ${ }^{(28)}$ mostraron además que la flexibilidad psicológica posibilitó la puesta en marcha de comportamientos congruentes con valores personales, lo cual estuvo en sí mismo asociado a mayores niveles de bienestar. A modo general, estos estudios muestran que la flexibilidad psicológica puede tener un amplio abanico de efectos positivos sobre diversos indicadores de bienestar en personas con cáncer, lo cual apunta a su relevancia como objetivo del trabajo terapéutico en psicooncología.

De hecho, algunos estudios de intervención han dado buena cuenta de que el trabajo terapéutico destinado a promover la flexibilidad psicológica es de gran relevancia en el contexto del cáncer. Aguirre-Camacho et al. ${ }^{(29)}$ mostraron que la inflexibilidad psicológica parece perpetuar los problemas emocionales y atenuar los efectos positivos de las intervenciones terapéuticas. Dicho estudio, en el que participaron 54 mujeres con cáncer de mama, consistió en una intervención de tipo cognitivo-conductual de 10 sesiones de duración, en la que no se incluyeron componentes para promover la flexibilidad psicológica. La participación en la intervención estuvo asociada a cambios significativos pre-post en los niveles de ansiedad y depresión, pero no en flexibilidad psicológica. Sin embargo, la inflexibilidad psicológica estuvo asociada con niveles más altos de ansiedad y depresión al finalizar la intervención. Los autores sugieren que las terapias de tipo cognitivo-conductual podrían no ser efectivas en aminorar la inflexibilidad psicológica, pero que en cambio, la inflexibilidad psicológica sí podría interferir con las metas terapéuticas planteadas por este tipo de terapias. Este aspecto es de gran relevancia dado que las terapias de tipo cognitivo conductual son quizás las más empleadas actualmente en la práctica clínica en psico-oncología.

Hasta el momento han sido pocos los estudios en psico-oncología que han evaluado la efectividad de intervenciones basadas en ACT, las cuales precisamente tienen entre sus objetivos principales la promoción de la flexibilidad psicológica. Sin embargo, los estudios realizados han mostrado de forma consistente que el incremento de la flexibilidad psicológica está asociado a mejores resultados terapéuticos. El primer trabajo en esta área fue publicado por Montesinos Marin et al. en el año $2001^{(30)}$, y corresponde a un estudio de caso de aplicación de ACT a un hombre de 50 años diagnosticado con cáncer de mama. La intervención tuvo una duración de 20 sesiones, y estuvo asociada a reducciones en los niveles de evitación experiencial, ansiedad, y depresión. Karekla y Constantinou ${ }^{(31)}$ publicaron también otro estudio de caso, en el que se describe la aplicación de ACT a una mujer de 58 años diagnosticada con cáncer de mama y en fase de tratamiento con quimioterapia. En este estudio se hace especial énfasis en la relevancia de los valores personales en el trabajo terapéutico dirigido a promover la flexibilidad psicológica con el fin de dirigir la conducta hacia fines valiosos. Específicamente, la terapia estuvo centrada en dos valores de gran importancia para la paciente, su religión y el amor a sus nietas, los cuales le sirvieron de brújula en su intento de reencontrar un sentido de dirección en su vida. Sin duda, los estudios de caso pueden 
aportar información muy valiosa y detallada desde una perspectiva ideográfica, pero presentan limitaciones debido a su baja validez externa.

La efectividad de las intervenciones basadas en ACT en esta área ha sido evaluada en varios ensayos clínicos aleatorizados (ECA); al menos tres de ellos han sido específicamente llevados a cabo con mujeres con cáncer de mama. El primero de éstos fue realizado por Páez et al. ${ }^{(32)}$ y utilizó una muestra de 12 mujeres, las cuales fueron asignadas a un grupo basado en el trabajo en la aceptación y otro grupo basado en el trabajo en el control cognitivo. Los resultados mostraron que a pesar de que las reducciones en los niveles de ansiedad y depresión e incrementos en calidad de vida fueron equivalentes en los dos grupos al terminar la intervención, las participantes en el grupo de aceptación mostraron mayores mejorías en las tres variables al cabo de 12 meses. Además, los resultados mostraron que las participantes en el grupo de aceptación mostraron una mayor activación conductual y mejor capacidad de redirigir sus vidas hacia fines valiosos, aun en presencia de sentimientos y pensamientos aversivos. Resultados similares fueron obtenidos en otros dos ECA recientemente realizados en Irán, en los cuales ACT fue comparada con un grupo control. Najvani et al. ${ }^{(33)}$, empleando una nuestra de 16 pacientes, mostraron que ACT generó reducciones en los niveles de depresión e incrementos en flexibilidad psicológica; por su parte, Mohabbat-Bahar et al. ${ }^{(34)}$, utilizando una muestra de 30 participantes, mostraron que ACT resultó en reducciones en los niveles de ansiedad y depresión. Aún más recientemente, Montesinos y Luciano ${ }^{(35)}$ realizaron un ensayo controlado no aleatorio dirigido a disminuir los niveles de miedo a la recidiva, en el que 12 mujeres con cáncer de mama fueron asignadas a ACT o lista de espera. La intervención contó con una sola sesión, que incluyo componentes dirigidos a la clarificación de valores, a la aceptación del miedo a la recidiva, y a disminuir el nivel de fusión cognitiva con pensamientos acerca del cáncer. Los resultados mostraron que ACT generó reducciones significativas en el nivel de interferencia causada por el miedo a la recidiva, lo cual resulta alentador dada la brevedad de la intervención. En comparación con los estudios de caso descritos anteriormente, estos tres estudios ofrecen evidencia más robusta sobre la efectividad de ACT en el trabajo terapéutico con pacientes con cáncer. Los diseños con grupo control y la aleatorización constituyen una fortaleza, si bien las relativas pequeñas muestras constituyen una limitación.

La efectividad de ACT en el contexto del cáncer fue también evaluada comparándola con una intervención basada en terapia cognitivo-conductual (TCC), como parte de un ECA para mujeres con cáncer de ovarios en estado avanzado ${ }^{(36)}$. Este ECA contó con una muestra mayor $(\mathrm{N}=47)$ en comparación con los ECA descritos anteriormente, pero aún más importante, como parte de su diseño se realizaron análisis de mediación con el objetivo de examinar los procesos de cambio durante el transcurso de la intervención y el seguimiento. Los resultados al finalizar la intervención mostraron que las participantes que recibieron $\mathrm{ACT}$, en comparación con TCC, mostraron mayores disminuciones en los niveles de distrés, ansiedad, y depresión, así como mayores incrementos en calidad de vida. Además, los análisis de mediación mostraron que los cambios en el grupo ACT fueron el resultado de los incrementos observados en los niveles de flexibilidad psicológica. Por el contrario, los niveles de flexibilidad psicológica disminuyeron en el grupo que recibió TCC, lo cual según los autores parece haber sido el resultado de declives en el estado físico en ausencia de entrenamiento en flexibilidad psicológica. Es de notar que las reducciones en los niveles de ansiedad y depresión fueron menores en este grupo 
en comparación con el grupo que recibió ACT, a pesar de que las intervenciones basadas en TCC cuentan con componentes destinados a tratar la ansiedad y depresión.

La relevancia de la flexibilidad psicológica como variable mediadora de los efectos de ACT en el bienestar de pacientes con cáncer fue constatada por otros dos estudios. Uno de estos estudios fue realizado por Feros et al. ${ }^{(37)}$ e incluyó una muestra de 45 pacientes con diferentes tipos de cáncer, los cuales fueron evaluados al comienzo, después de la cuarta sesión, al final de la intervención, y tres meses después. En el transcurso de nueve sesiones individuales los participantes mostraron reducciones en los niveles de distrés y de alteraciones del estado de ánimo e incrementos en la calidad de vida, lo cual se mantuvo al cabo de tres meses. Además, los resultados mostraron que a partir de la mitad de la intervención el cambio en estas variables ocurrió de forma posterior a los incrementos en flexibilidad psicológica. Similares resultados fueron obtenidos por Arch y Mitchell ${ }^{(38)}$ en una intervención destinada a 42 pacientes con diferentes tipos de cáncer que se encontraban en el proceso de transición hacia la fase de post-tratamiento. Este estudio incorporó como parte de su diseño tres evaluaciones en el mes que antecedió el comienzo de la intervención. Esto permitió evaluar posibles cambios e interacciones entre las variables durante el mes previo a la intervención, a modo de comparación con los cambios asociados con la participación en la intervención. Los incrementos observados en flexibilidad psicológica predijeron cambios positivos en diversas variables, tales como depresión, dolor físico, trauma relacionado con el cáncer, vitalidad, significado de la vida, y manejabilidad vital; también hubo una tendencia hacia cambios significativos en ansiedad y comprensión de la vida. Todos los resultados positivos fueron mantenidos después de 12 semanas. Los resultados de estos estudios son congruentes con muchos otros que han mostrado evidencia de que la flexibilidad psicológica constituye un importante mecanismo de cambio en $\mathrm{ACT}^{(7)}$. Además, estos estudios también sugieren que los cambios en flexibilidad psicológica parecen dar respuesta a buena parte de los problemas psicológicos de los pacientes con cáncer, dados los cambios positivos en diversos indicadores de bienestar.

La efectividad de ACT en el contexto del cáncer también ha sido apoyada en intervenciones de tipo no presencial. Hawkes et al. ${ }^{(39)}$ realizaron un ECA en el que 410 individuos sobrevivientes al cáncer de colon fueron aleatorizados a un grupo de tratamiento habitual o a una intervención basada en ACT; la intervención contó con un manual didáctico y consistió en 11 sesiones realizadas por teléfono durante un período de seis meses, destinadas a mejorar el manejo de los síntomas y del estrés y a promover estilos de vida saludables. En comparación con el tratamiento habitual, ACT dio lugar a incrementos en crecimiento post-traumático, espiritualidad, y flexibilidad psicológica. Además, la flexibilidad psicológica medió los cambios en crecimiento post-traumático, espiritualidad, y bienestar físico. Chambers et al. ${ }^{(40)}$ también realizaron una intervención por teléfono que contó con un total de seis sesiones semanales dirigida a 14 pacientes con cáncer de pulmón. La intervención fue de tipo cognitivo-conductual pero incorporó elementos de ACT, y también incluyó material didáctico. Los resultados mostraron que la participación en la intervención estuvo asociada a reducciones en los niveles de depresión y estigma relacionado con el cáncer de pulmón. Estos resultados fueron obtenidos a pesar de que los participantes sufrieron deterioros en la calidad de vida durante el transcurso de la intervención, lo cual sugiere que las intervenciones dirigidas a promover la 
flexibilidad psicológica podrían atenuar el impacto del deterioro de la salud en el bienestar emocional de pacientes con cáncer. Un aspecto positivo y novedoso de esta intervención fue la integración de la TCC con componentes de ACT, con el fin de contar con una intervención mejor adaptada a las necesidades de los pacientes. Estos dos estudios muestran que las intervenciones basadas en ACT pueden ser adaptadas de forma efectiva a pacientes con dificultades en el acceso a centros de salud.

\section{Discusión e implicaciones para la práctica clínica}

La flexibilidad psicológica es un constructo de gran relevancia para la investigación y la práctica clínica en psico-oncología. La investigación sobre flexibilidad psicológica en el contexto del cáncer no es extensa, pero muestra resultados consistentes: la flexibilidad psicológica se asocia positivamente con un buen número de indicadores de bienestar en pacientes con cáncer y constituye un mecanismo de cambio en el trabajo terapéutico con pacientes de cáncer. La atención destinada a la flexibilidad psicológica dentro de la psico-oncología ha sido notablemente menor que en otras áreas, pero parece estar aumentando a buen ritmo: más de la mitad de los estudios que fueron identificados y presentados en esta revisión fueron publicados en los últimos dos años.

El trabajo terapéutico destinado a incrementar la flexibilidad psicológica en pacientes con cáncer, según se implementa en ACT, puede centrarse en diferentes objetivos $^{(41)}$. Ya se ha visto por ejemplo que el trabajo destinado a disminuir la fusión cognitiva con pensamientos relacionados con el cáncer puede dar lugar a disminuciones de los niveles de miedo a recidiva en mujeres con cáncer de mama ${ }^{(35)}$, y de igual forma podría ser aplicado en pacientes con otros tipos de cáncer. Tal y como se ha expuesto anteriormente, el trabajo destinado a disminuir los niveles de fusión cognitiva puede además ser de gran utilidad con vistas a disminuir la evitación experiencial, o lo que es lo mismo, a incrementar los niveles de aceptación.

El incremento de los niveles de aceptación puede ser de gran relevancia en el contexto del cáncer por varios motivos. En primer lugar, nuestro contexto cultural no nos prepara para aceptar, sino para luchar; a los pacientes de cáncer se les suele decir que es importante desarrollar un "espíritu de lucha" para poder ganar "la batalla contra el cáncer". A pesar de que dichos estímulos verbales pueden generar conductas funcionales cuando motivan a los pacientes a buscar atención médica y apoyo social, éstos también pueden conducir a un comportamiento altamente disfuncional cuando la "guerra contra el cáncer" también se convierte en la guerra contra eventos privados relacionados con el cáncer (i.e. cuando se intentan reprimir pensamientos o sentimientos). Además de inefectiva, dicha guerra puede provocar al menos dos consecuencias negativas. En primer lugar, tal y como se ha visto, puede llevar a incrementos en la frecuencia e intensidad de los mismos eventos privados que tratan de evitarse ${ }^{(24)}$. En segundo lugar, la guerra contra los eventos privados relacionados con el cáncer puede interferir con la puesta en marcha de conductas congruentes con los valores personales ${ }^{(28,31,32)}$. Y es que implícitamente, dicha guerra es en cierto sentido impulsada por la fusión cognitiva con la creencia de que "se debe estar totalmente bien" antes de poder dedicarse a fines valiosos.

Precisamente, el trabajo terapéutico dirigido a contactar con los valores personales y dirigir la conducta hacia dichos valores, aún en presencia de malestar, es otro de 
los objetivos centrales de $\mathrm{ACT}^{(7)}$. En sí mismo, se ha visto que la realización de acciones congruentes con los valores personales puede incrementar la sensación de bienestar en pacientes con cáncer ${ }^{(28,31)}$. Al mismo tiempo, el incremento de la actividad conductual y la realización de actividades placenteras pueden ayudar a disminuir la presencia del cáncer en la vida de los pacientes, y por lo tanto, los niveles de afecto negativo ${ }^{(32)}$.

Las aplicaciones terapéuticas destinadas a incrementar los niveles de flexibilidad psicológica han sido ampliamente desarrolladas como parte de ACT. Sin embargo, al menos un estudio ha aportado evidencia de que algunos de los componentes de ACT podrían ser integrados de forma exitosa como parte de las intervenciones basadas en $\mathrm{TCC}^{(40)}$. En algunos casos, puede que dicha integración pueda potenciar los resultados de las intervenciones basadas en $\mathrm{TCC}^{(29)}$. En cualquier caso, cada vez es mayor el número de estudios que aportan evidencia no solo sobre la efectividad de las terapias psicológicas en su totalidad, sino también sobre los distintos componentes de dichas terapias que constituyen mecanismos de cambio. Por lo tanto, será la labor del personal clínico determinar qué componentes podrían ser efectivos para hacer frente a determinado tipo de problemas presentes en determinados pacientes.

\section{Referencias bibliográficas}

1. Singer S, Das-Munshi J, Brähler E. Prevalence of mental health conditions in cancer patients in acute care - a meta-analysis. Ann Oncol 2010;21:925-930. Doi:10.1093/ annonc/mdp515.

2. Gottlieb N. The age of breast cancer awareness: what is the effect of media coverage? J Natl Cancer Inst 2001;93:1520-1522. Doi:10.1093/jnci/93.20.1520

3. Georges J, Benson JM, Wikler EW, Weldon KJ, Baumgart M, Jansen S. Key Findings from a five-country survey of public attitudes about Alzheimer's disease. Poster presented at AAIC. 2011 Jul.

4. Camacho AA, Garland SN, Martopullo C, Pelletier G. Positive and negative meanings are simultaneously ascribed to colorectal cancer: relationship to quality of life and psychosocial adjustment. Palliat Support Care 2014 1;12:277-86. Doi: 10.1017/S1478951513000151

5. Niederdeppe J, Levy AG. Fatalistic beliefs about cancer prevention and three prevention behaviors. Cancer Epidemiol Biomarkers Prev 2007;16:998-1003. Doi: 10.1158/10559965.EPI-06-0608

6. Bond FW, Hayes SC, Baer RA, Carpenter KM, Guenole N, Orcutt HK, et al. Preliminary psychometric properties of the Acceptance and Action Questionnaire-II: A revised measure of psychological inflexibility and experiential avoidance. Behav Ther 2011;42:676-88. Doi: 10.1016/j.beth.2011.03.007

7. Hayes SC, Strosahl KD, Wilson KG. Acceptance and commitment therapy: The process and practice of mindful change. Guilford Press; 2011.

8. Kashdan TB, Barrios V, Forsyth JP, Steger MF. Experiential avoidance as a generalized psychological vulnerability: Comparisons with coping and emotion regulation strategies. Behav Res Ther 2006;44:1301-20.

9. Roemer L, Salters K, Raffa SD, Orsillo SM. Fear and avoidance of internal experiences in GAD: Preliminary tests of a conceptual model. Cognit Ther Res 2005;29:71-88. 
10. Tull MT, Gratz KL, Salters K, Roemer L. The role of experiential avoidance in posttraumatic stress symptoms and symptoms of depression, anxiety, and somatization. $\mathrm{J}$ Nerv Mental Dis 2004;192:754-61.

11. Cribb G, Moulds ML, Carter S. Rumination and experiential avoidance in depression. Behav Change 2006;23:165-76. Doi:10.1375/bech.23.3.165

12. Goodwin CL, Forman EM, Herbert JD, Butryn ML, Ledley GS. A pilot study examining the initial effectiveness of a brief acceptance-based behavior therapy for modifying diet and physical activity among cardiac patients. Behav Mod 2012;36:199-217. Doi: 10.1177/0145445511427770

13. Nordin L, Rorsman I. Cognitive behavioural therapy in multiple sclerosis: a randomized controlled pilot study of acceptance and commitment therapy. J Rehab Med 2012;44:8790. Doi: 10.2340/16501977-0898.

14. Hulbert-Williams NJ, Storey L, Wilson KG. Psychological interventions for patients with cancer: psychological flexibility and the potential utility of Acceptance and Commitment Therapy. Eur J Cancer Care 2015;24:15-27. Doi: 10.1111/ecc.12223

15. Brown P, Zavestoski SM, McCormick S, Mandelbaum J, Luebke T. Print media coverage of environmental causation of breast cancer. Sociol Health Illn 2001;23:747-75.

16. Blanchard D, Erblich J, Montgomery GH, Bovbjerg DH. Read all about it: the overrepresentation of breast cancer in popular magazines. Prev Med 2002;35:343-8. Doi: 10.1006/pmed.2002.1088

17. Dutta-Bergman MJ. Primary sources of health information: Comparisons in the domain of health attitudes, health cognitions, and health behaviors. Health comm 2004;16:273-88.

18. Rees CE, Bath PA. Mass media sources for breast cancer information: their advantages and disadvantages for women with the disease. JDoc. 2000;56:235-49. Doi:10.1108/ EUM0000000007114

19. Bray F, McCarron P, Parkin DM. The changing global patterns of female breast cancer incidence and mortality. Breast Cancer Res 2004;6:229. Doi: 10.1186/bcr932

20. Clarke JN, Everest MM. Cancer in the mass print media: Fear, uncertainty and the medical model. Soc Sci Med 2006;62:2591-600. Doi:10.1016/j.socscimed.2005.11.021

21. DiLorenzo TA, Schnur J, Montgomery GH, Erblich J, Winkel G, Bovbjerg DH. A model of disease-specific worry in heritable disease: the influence of family history, perceived risk and worry about other illnesses. J Behav Med 2006;29:37-49. Doi: 10.1007/s10865005-9039-y

22. Wang C, O’Neill SM, Rothrock N, Gramling R, Sen A, Acheson LS, et al. Comparison of risk perceptions and beliefs across common chronic diseases. Prev Med 2009;48:197202. Doi: 10.1016/j.ypmed.2008.11.008

23. Hall AG, Khoury AJ, Lopez ED, Lisovicz N, Avis-Williams A, Mitra A. Breast cancer fatalism: the role of women's perceptions of the health care system. J Health Care for the Poor and Underserved 2008;19:1321-35. Doi: 10.1353/hpu.0.0091.

24. Wegner DM. Ironic processes of mental control. Psychol Rev 1994;101:34-52.

25. Miller SJ, O'Hea EL, Lerner JB, Moon S, Foran-Tuller KA. The relationship between breast cancer anxiety and mammography: Experiential avoidance as a moderator. Behav Med 2011;37:113-8. Doi: 10.1080/08964289.2011.614291

26. Hulbert-Williams NJ, Storey L. Psychological flexibility correlates with patient-reported outcomes independent of clinical or sociodemographic characteristics. Suppor Care Cancer 2016;24:2513-21. Doi: 10.1007/s00520-015-3050-9 
27. Montiel CB, Moya TR, Venditti F, Bernini O. On the contribution of psychological flexibility to predict adjustment to breast cancer. Psicothema 2016;28:266-71. Doi: 10.7334/psicothema2015.271

28. Ciarrochi J, Fisher D, Lane L. The link between value motives, value success, and wellbeing among people diagnosed with cancer. Psychoncology 2011;20:1184-92. Doi: 10.1002 /pon. 1832

29. Aguirre-Camacho A, Pelletier G, González-Márquez A, Blanco-Donoso LM, GarcíaBorreguero P, Moreno-Jiménez B. The relevance of experiential avoidance in breast cancer distress: insights from a psychological group intervention. Psychooncology 2017;26:469-475. Doi: 10.1002/pon.4162

30. Montesinos Marín F, Hernández Montoyo B, Luciano Soriano MC. Aplicación de la Terapia de Aceptación y Compromiso en pacientes enfermos de cáncer. Análisis y Modificación de Conducta 2001;27:503-23.

31. Karekla M, Constantinou M. Religious coping and cancer: Proposing an acceptance and commitment therapy approach. Cogn Behav Practice 2010;17:371-81. Doi:10.1016/j. cbpra.2009.08.003

32. Páez MB, Luciano C, Gutiérrez O. Tratamiento psicológico para el afrontamiento del cáncer de mama. Estudio comparativo entre estrategias de aceptación y de control cognitivo. Psicooncología 2007;4:75-95.

33. Najvani BD, Neshatdoost HT, Abedi MR, Mokarian F. The effect of acceptance and commitment therapy on depression and psychological flexibility in women with breast cancer. Zahedan J Res Med Sci 2015;17. Doi:10.17795/zjrms965

34. Mohabbat-Bahar S, Maleki-Rizi F, Akbari ME, Moradi-Joo M. Effectiveness of group training based on acceptance and commitment therapy on anxiety and depression of women with breast cancer. Iran J Cancer Prev 2015;8:71.

35. Montesinos F, Luciano C. Acceptance of relapse fears in breast cancer patients: Effects of an ACT-based abridged intervention. Psicooncología 2016;13:7-21. Doi:10.5209/rev_ PSIC.2016.v13.n1.52484

36. Rost AD, Wilson K, Buchanan E, Hildebrandt MJ, Mutch D. Improving psychological adjustment among late-stage ovarian cancer patients: examining the role of avoidance in treatment. Cogn Behav Pract 2012;19:508-17. Doi:10.1016/j.cbpra.2012.01.003

37. Feros DL, Lane L, Ciarrochi J, Blackledge JT. Acceptance and Commitment Therapy (ACT) for improving the lives of cancer patients: A preliminary study. Psychooncology 2013;22:459-64. Doi: 10.1002/pon.2083

38. Arch JJ, Mitchell JL. An Acceptance and Commitment Therapy (ACT) group intervention for cancer survivors experiencing anxiety at re-entry. Psycho-oncology. 2016;25:610-5. Doi: 10.1002/pon.3890

39. Hawkes AL, Pakenham KI, Chambers SK, Patrao TA, Courneya KS. Effects of a multiple health behavior change intervention for colorectal cancer survivors on psychosocial outcomes and quality of life: a randomized controlled trial. Ann Behav Med 201448:35970. Doi: 10.1007/s12160-014-9610-2.

40. Chambers SK, Morris BA, Clutton S, Foley E, Giles L, Schofield P, et al. Psychological wellness and health-related stigma: a pilot study of an acceptance-focused cognitive behavioural intervention for people with lung cancer. Eur J Cancer Care 2015;24:60-70. Doi: $10.1111 /$ ecc. 12221

41. Páez MB, Luciano C, Gutierrez O. La aplicación de la Terapia de Aceptación y Compromiso (ACT) en el tratamiento de problemas psicológicos asociados al cáncer. Psicooncología 2005;2:49-70. 